\title{
Mortality Effects of Prolonged Hemoperfusion Therapy Using a Polymyxin B-Immobilized Fiber Column for Patients with Septic Shock: A Sub-Analysis of the DESIRE Trial
}

\author{
Yu Kawazoe $^{a}$ Tetsuya Sato $^{\text {b }}$ Noriko Miyagawa $^{\mathrm{b}}$ Yuta Yokokawa $^{\mathrm{b}}$ \\ Shigeki Kushimoto $^{a}$ Kyohei Miyamoto $^{c}$ Yoshinori Ohtad $^{d}$ Takeshi Morimoto $^{\mathrm{e}}$ \\ Hitoshi Yamamura $^{f}$ \\ ${ }^{a}$ Division of Emergency and Critical Care Medicine, Tohoku University Graduate School of Medicine, Sendai, Japan; \\ ${ }^{b}$ Department of Emergency and Critical Care Medicine, Tohoku University Hospital, Sendai, Japan; ' Department \\ of Emergency and Critical Care Medicine, Wakayama Medical University, Wakayama, Japan; ${ }^{\mathrm{d} D i v i s i o n}$ of General \\ Medicine, Department of Internal Medicine, Hyogo College of Medicine, Nishinomiya, Japan; ${ }^{e}$ Department of \\ Clinical Epidemiology, Hyogo College of Medicine, Nishinomiya, Japan; ${ }^{\mathrm{D}}$ Department of Disaster and Critical Care \\ Medicine, Hirosaki University Graduate School of Medicine, Hirosaki, Japan
}

\section{Keywords}

Endotoxin · Hemoperfusion therapy · Optimal duration ·

Septic shock · Toraymyxin

\begin{abstract}
Background/Aims: The optimal duration of hemoperfusion therapy with a polymyxin B-immobilized fiber column has not yet been verified. Methods: This analysis examined whether hemoperfusion therapy with a polymyxin B-immobilized fiber column lasting longer than $2 \mathrm{~h}$ (prolonged polymyxin) improved outcomes for patients with septic shock compared to 2-h polymyxin therapy (sub-analysis of data from the DESIRE trial). Results: The 2-h and prolonged polymyxin groups contained 22 and 14 patients, respectively. Both groups had similar characteristics. The polymyxin duration per session in the prolonged polymyxin group was significantly longer (median, $5.5 \mathrm{~h}$ ) than in the 2-h polymyxin group ( $p<0.01)$. The 28 -day mortality rate was significantly higher in the 2 -h polymyxin group $(7,31.8 \%)$ than in the pro-
\end{abstract}

๑) 2018 S. Karger AG, Basel

E-Mail karger@karger.com www.karger.com/bpu longed polymyxin group $(0,0 \% ; p=0.019)$. Conclusion: Prolonged polymyxin therapy might be associated with better clinical outcomes than 2-h polymyxin therapy in patients with septic shock. Video Journal Club "Cappuccino with Claudio Ronco" at http://www.karger.com/?doi=491744.

(c) 2018 S. Karger AG, Basel

\section{Introduction}

Septic shock in intensive care units (ICUs) is associated with poor prognosis $[1,2]$. Various humoral mediators, including inflammatory cytokines, have been demonstrated to be involved in the pathogenesis of septic shock and sepsis-associated organ dysfunction. Blood purification techniques have been expected to improve patients' condition and outcome by removing pathologic mediators [3-6]. Hemoperfusion therapy using a polymyxin B-immobilized fiber column (PMX-HP) has been proposed as adjunctive therapy to remove circulating en- 
dotoxins derived from the outer membrane of Gramnegative bacteria. These endotoxins are typical pathogenassociated molecules present in patients with sepsis. Meta-analyses revealed that PMX-HP was associated with improved outcomes (e.g., improved hemodynamics, respiratory status, and mortality) $[7,8]$. However, no beneficial effect was detected in a recent randomized controlled trial (RCT) [9]. Thus, it remains unclear whether conventional PMX-HP can improve outcomes in septic shock.

In Japan, PMX-HP (Toraymyxin ${ }^{\circledR}$ Cartridge, Toray Medical, Tokyo, Japan) for patients with sepsis is approved by health insurance schemes. Sepsis is treated in line with the Japanese guideline for the management of sepsis [10], but the appropriate duration of PMX-HP for each case is not indicated. Two hours of PMX-HP was performed in the previous RCTs; however, some researchers assumed that prolonged PMX-HP could be effective especially for patients with refractory shock. Therefore, in Japan, some physicians use the PMX-HP longer than $2 \mathrm{~h}$ at the discretion of the attending physician.

The present study investigated whether prolonged PMX-HP was associated with more favorable outcomes among patients with septic shock compared to the conventional 2-h PMX-HP regimen. Data from the Dexmedetomidine for Sepsis in the ICU Randomized Evaluation (DESIRE) trial [11] were evaluated.

\section{Materials and Methods}

Ethical Considerations and Patient Selection

This nested cohort analysis examined data from the DESIRE trial [11], which was a multicenter (8 Japanese ICUs) RCT that studied 201 patients with sepsis requiring ventilation. The DESIRE trial protocol has been reported elsewhere [11], and ethical approval was received from all relevant institutional review boards and all participants provided informed consent prior to enrolment. The present study evaluated data from patients who presented with septic shock, defined as a score of $\geq 3$ for the cardiovascular components of the Sequential Organ Failure Assessment and serum lactate levels of $>2.0 \mathrm{mmol} / \mathrm{L}$. To achieve this score, the patients would have required a moderate or high dose of vasopressors; therefore, they were also defined as having septic shock according to the Sepsis III criteria.

\section{Patient Management}

In this study, patients were categorized into 2 groups according to whether they received the conventional 2-h PMX-HP or prolonged ( $>2$ h) PMX-HP during their first session. Subsequent use was not considered during patient categorization.

\section{Data Collection}

We collected patient data on age and sex, as well as initial serum lactate levels, day-1 Acute Physiology and Chronic Health Evaluation II (APACHE II) scores, Sequential Organ Failure Assessment scores, C-reactive protein levels, and procalcitonin levels. In addition, we collected data regarding the duration of PMX-HP at the first session, PMX-HP use on day 2, use of renal replacement therapy, infection site (abdomen, thorax, or other), 28-day mortality, in-hospital mortality, vasopressor use (i.e., noradrenaline or vasopressin), steroid use for shock, and ventilator-free days during the first 28 days. We also collected reference data for a group of patients with septic shock who did not receive PMX-HP. The primary outcome was 28-day mortality, and secondary outcomes were in-hospital mortality, vasopressor-free days during the first 7 days (alive and without vasopressor use), and ventilator-free days during the first 28 days.

\section{Statistical Analysis}

Continuous variables are reported as means \pm SDs or medians and interquartile ranges. Categorical variables are reported as numbers and percentages. Categorical variables were compared using a chi-square test or Fisher's exact test, and continuous variables were compared using the $t$ test or Wilcoxon rank sum test, based on the normality of variable distribution. Although we had planned to use a Cox proportional hazard model and a multivariate logistic regression model, adjusted for age, APACHE II score, and dexmedetomidine use, these analyses were not performed due to over-adjustment. Two-sided $p$ values $<0.05$ were considered statistically significant. All analyses were performed using JMP Pro software (version 12.2; SAS Institute, Cary, NC, USA).

\section{Results}

Among the 201 patients enrolled in the DESIRE trial, this study focused on the 112 patients with septic shock. Of these patients, 36 received PMX-HP, and 76 did not (online suppl. Table 1, see www.karger.com/ doi/10.1159/000491744). Non-significant differences in APACHE II scores, infection sites, procalcitonin levels, renal replacement therapy use, and vasopressin use were observed between patients receiving and not receiving PMX-HP.

Of the patients receiving PMX-HP, 22 received conventional 2-h PMX-HP and 14 received prolonged PMX-HP (Fig. 1). Patients in both groups had similar characteristics (Table 1), except for the total duration of PMX-HP. Patients in the 2-h PMX-HP group received PMX-HP for $2 \mathrm{~h}$, whereas those in the prolonged PMXHP group received PMX-HP for a median of $5.5 \mathrm{~h}$ (interquartile range $3.8-12.50)$. Treatment durations were significantly different $(p<0.01)$. More patients in the $2-\mathrm{h}$ PMX-HP group (63.6\%) than in the prolonged PMX-HP group $(21.4 \%)$ underwent PMX-HP on day $2(p=0.013)$. There was no significant difference between groups re- 
Fig. 1. Patient selection flowchart. DESIRE, Dexmedetomidine for Sepsis in the Intensive care unit (ICU) Randomized Evaluation; PMX-HP, hemoperfusion therapy using a polymyxin B-immobilized fiber column.

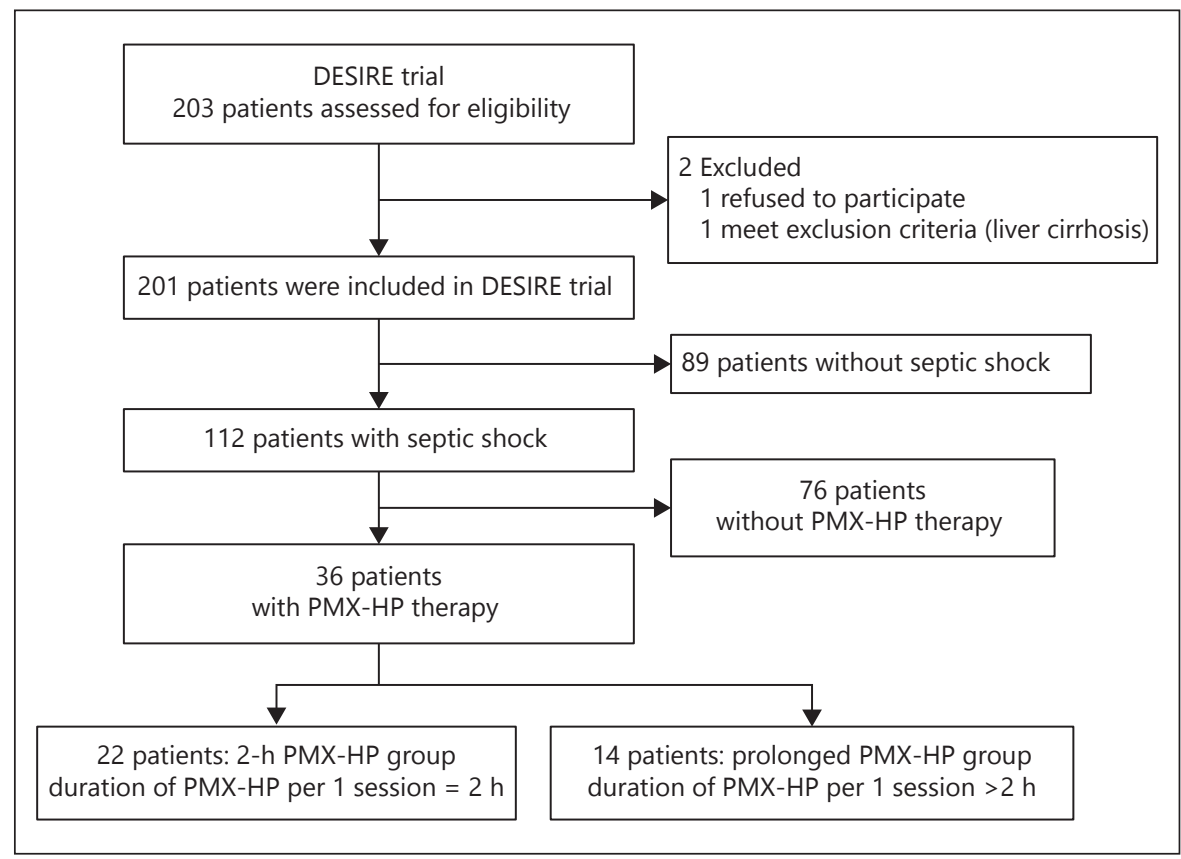

Table 1. Patient characteristics

\begin{tabular}{lccc}
\hline & $\begin{array}{c}\text { 2-h PMX-HP } \\
\text { group }(n=22)\end{array}$ & $\begin{array}{c}\text { Prolonged PMX-HP } \\
\text { group }(n=14)\end{array}$ & $p$ value \\
\hline Age, years & $73.8 \pm 14.0$ & $74.8 \pm 8.6$ & 0.82 \\
Gender, male & $13(59.1)$ & $7(50.0)$ & 0.59 \\
APACHE II score ${ }^{\dagger}$ & $24.5(19-29.3)$ & $22.5(18-29.5)$ & 0.57 \\
Lactate, mmol/L & $5.1(3.8-8.3)$ & $3.8(2.8-5.5)$ & 0.12 \\
Treatment using dexmedetomidine & $13(59.1)$ & $5(42.9)$ & 0.34 \\
Day 1 SOFA score ${ }^{\ddagger}$ & $10.5(8-12)$ & $11(7-11.5)$ & 0.79 \\
Day 1 C-reactive protein, mg/dL & $6.5(2.2-21.4)$ & $17.5(2.2-31.9)$ & 0.24 \\
Day 1 procalcitonin, ng/mL & $44.9(28.8-97.5)$ & $45.4(22.2-154.0)$ & 0.92 \\
Duration of PMX-HP per session, h & $2.0(2.0-2.0)$ & $5.5(3.75-12.5)$ & $<0.01$ \\
PMX-HP on the second day & $14(63.6)$ & $3(21.4)$ & 0.013 \\
Renal replacement therapy & $11(50)$ & $10(71.4)$ & 0.20 \\
Site of infection & & $9(64.3)$ & 0.97 \\
$\quad$ Abdomen & $14(63.6)$ & $1(7.1)$ & 0.84 \\
$\quad$ Thorax & $2(9.1)$ & $4(28.6)$ & 0.93 \\
$\quad$ Other & $6(27.3)$ & & 0.34 \\
Type of pathogen & & $6(42.9)$ & 1.0 \\
$\quad$ Gram-negative bacteria & $13(59.1)$ & $3(21.4)$ & 0.46 \\
Gram-positive bacteria & $5(22.7)$ & $5(35.7)$ & 1.00 \\
Culture-negative & $5(22.7)$ & $14(100)$ & 0.40 \\
Vasopressors & & $5(35.7)$ & 0.54 \\
$\quad$ Noradrenaline & $22(100)$ & $11(78.6)$ & \\
Vasopressin & $5(22.7)$ & & \\
Steroids & $19(86.4)$ & & \\
\hline & & & \\
\hline
\end{tabular}

Data are reported as means \pm SDs, medians (interquartile ranges), or numbers (\%).

$\dagger$ APACHE II scores range from 0 to 71 , with higher scores indicating more severe disease.

‡ SOFA scores range from 0 to 24, with higher scores indicating more severe disease.

APACHE II, Acute Physiology and Chronic Health Evaluation II; SOFA, Sequential Organ Failure Assessment; PMX-HP, hemoperfusion therapy using a polymyxin B-immobilized fiber column. 
Fig. 2. Kaplan-Meier survival curves for mortality in patients in the 2 -h and prolonged PMX-HP groups. The numbers of patients still at risk of death are indicated in the figure. The criteria for patients at risk was patients alive and in hospital on the day. PMX-HP, hemoperfusion therapy using a polymyxin B-immobilized fiber column.

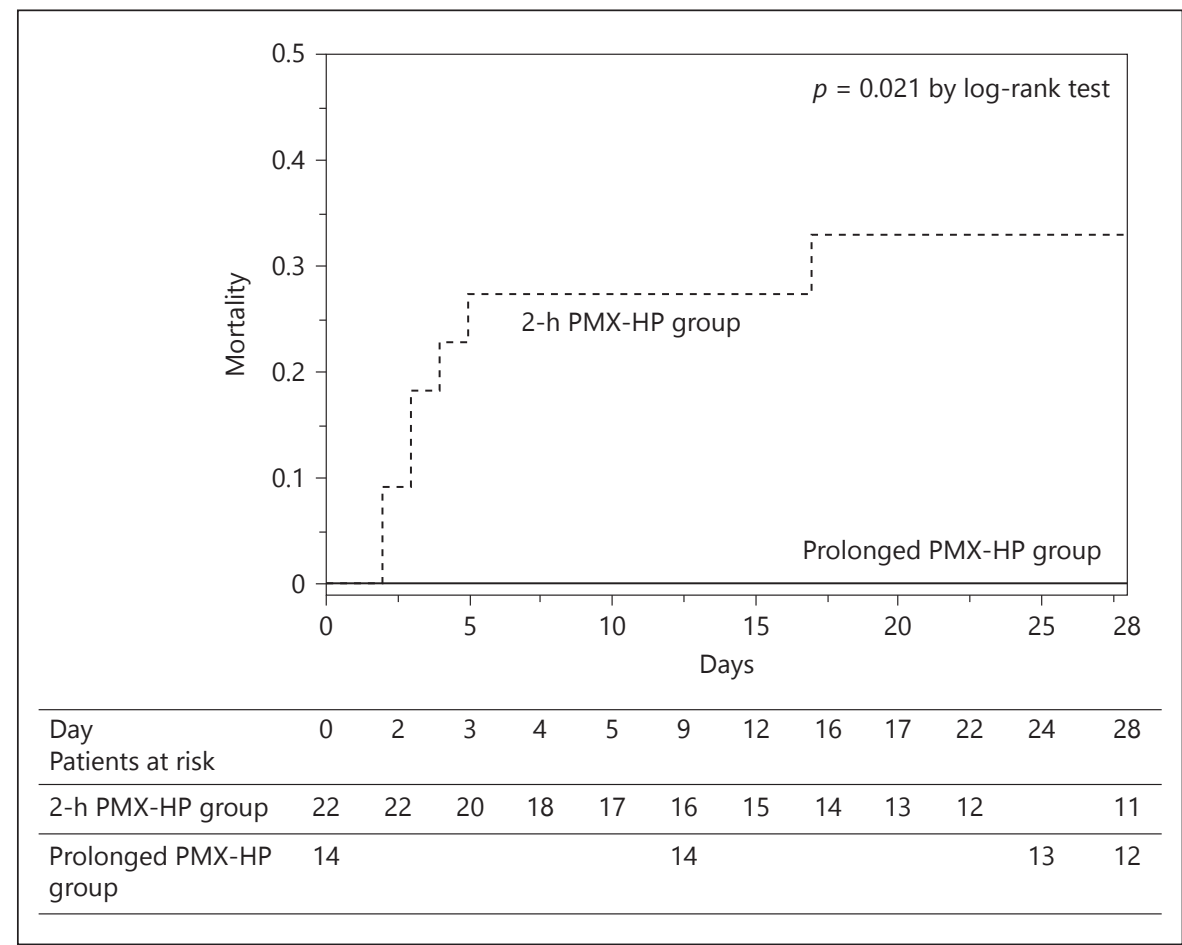

Table 2. Primary and secondary outcomes

\begin{tabular}{lccc}
\hline & $\begin{array}{l}\text { 2-h PMX-HP } \\
\text { group }(n=22)\end{array}$ & $\begin{array}{l}\text { Prolonged PMX-HP } \\
\text { group }(n=14)\end{array}$ & $p$ value \\
\hline 28-Day mortality & $7(31.8)$ & $0(0)$ & 0.019 \\
Ventilator-free days during initial 28 days & $11.5(10.5)$ & $15.6(8.3)$ & 0.23 \\
Vasopressor-free days during initial 7 days & $2.5(0.46)$ & $3.1(0.58)$ & 0.41
\end{tabular}

Data are reported as numbers (\%).

PMX-HP, hemoperfusion therapy using a polymyxin B-immobilized fiber column.

garding the proportions of the types of pathogens. Gramnegative bacteria were detected in 59.1 and $42.9 \%$ of cases, while Gram-positive bacteria were detected in 22.7 and $21.4 \%$ of cases in the 2-h PMX-HP group and in the prolonged PMX-HP group, respectively. No pathogens were identified in 22.7 and $35.7 \%$ of cases in each group $(p=0.46)$.

The 28-day mortality rate was significantly higher in the 2-h PMX-HP group $(7,31.8 \%)$ than in the prolonged PMX-HP group ( $0,0 \% ; p=0.019 ;$ Fig. 2$)$. There was no significant difference between the groups in vasopressor-free days during the first 7 days $(p=0.41)$ or in ventilator-free days during the first 28 days $(p=0.23$; Table 2).

\section{Discussion}

Our post-hoc analysis of DESIRE trial patients with septic shock who underwent PMX-HP suggested that prolonged PMX-HP might be associated with improved clinical outcomes than the conventional 2-h PMX-HP. Our results suggest that the effect of longer PMX-HP may be worthy of further clinical evaluation including RCTs.

PMX-HP is a unique therapy developed and widespread in Japan after 1994 that removes circulating endotoxins. Although systematic reviews concluded that PMX-HP therapy was associated with reduced mortality among patients with sepsis [7, 12], these findings were potentially biased by the increased likelihood that positive 
results were reported and the relatively low quality of the included studies. Subsequently, the updated meta-analysis by Chang et al. [8] concluded that PMX-HP improved survival among intermediate-risk and high-risk subgroups of patients with sepsis and septic shock. Two RCTs have also compared conventional 2-h PMX-HP with standard therapy among patients with severe abdominal sepsis and septic shock $[9,13]$. However, both RCTs failed to demonstrate that PMX-HP contributed to reduced mortality. Therefore, whether PMX-HP positively contributes to the treatment of septic shock patients remains unclear.

We believe that a key point of this treatment may be the duration of 1 session and not the frequency based on clinical experiences, but investigations and discussions related to this point are insufficient to be conclusive. The duration of PMX-HP was initially set at $2 \mathrm{~h}$ according to an in vitro study of endotoxin adsorption by the PMX column [14]. In that particular study, $1,500 \mathrm{~mL}$ of $10 \mathrm{ng} /$ $\mathrm{mL}$ endotoxin solution $(>100 \times$ the normal clinical serum endotoxin concentration) was circulated through the column for $2 \mathrm{~h}$. The results indicated that most of the endotoxin was adsorbed within the first $30 \mathrm{~min}$. These results appear to have been misinterpreted to indicate that PMX$\mathrm{HP}$ works within $2 \mathrm{~h}$. The column is capable of absorbing up to $10,500 \mathrm{ng}$ of endotoxins, and the study did not indicate whether the clinical limit of absorption had been reached within the study time frame.

Therefore, some Japanese researchers have speculated that PMX-HP for $>2 \mathrm{~h}$ may be clinically effective, especially among patients with septic shock who are expected to experience prolonged high endotoxin levels. Mitaka et al. [15] demonstrated that PMX-HP removed endotoxins for at least $24 \mathrm{~h} \mathrm{[15]}$ and that prolonged PMX-HP might improve the pulmonary oxygenation associated with decreased endotoxins in septic shock [16]. Yamashita et al. [17] confirmed that prolonged PMX-HP improved hemodynamics and pulmonary oxygenation capacity among patients with severe sepsis and septic shock, and Miyamoto et al. [18] reported that 12-h PMX-HP provided superior sustained circulatory stabilization compared to that of 2-h PMX-HP. Therefore, some Japanese physicians use prolonged PMX-HP in their ICUs for patients with septic shock.

The present study has several limitations. The most critical limitation was that this study was a post-hoc analysis of a RCT. Therefore, the induction and duration of PMX-HP were not randomized. In addition, we could not construct multivariate models to adjust the differences in baseline characteristics due to the small numbers of patients and events. Although the 2 groups exhibited similar patient characteristics, many confounders may have existed. Therefore, our findings should be interpreted as a hypothesis-generating study. Second, the trial included patients with infections caused by pathogenic bacteria other than Gram-negative bacilli. Actually, more than $20 \%$ of patients were infected with Gram-positive cocci in both groups. However, some researchers reported that PMX-HP was also effective for the treatment of sepsis caused by Gram-positive cocci $[14,19]$. It is known that endotoxemia occurs not only in infections caused by Gram-negative bacilli but in the infections caused by Gram-positive cocci and Candida [20,21] and that toraymyxin absorbs anandamide and 2-arachidonylglycerol which have vasodilator action [22]. Actually, a considerable number of patients with sepsis caused by Gram-positive cocci had high endotoxin activity levels in the EUPHRATES trial [23]. Third, no patients died within 28 days in the prolonged PMX-HP group, which is unusual for patients with septic shock. Again, the number of patients was too small to conclude in this study.

\section{Conclusion}

Prolonged PMX-HP might be associated with better clinical outcomes among patients with septic shock, compared to the conventional 2-h PMX-HP. It is necessary to evaluate our result in further prospective studies.

\section{Acknowledgments}

We thank the DESIRE Trial investigators for their assistance, especially Tomonori Yamamoto (Osaka City University, Osaka, Japan), Akihiro Fuke (Osaka City General Hospital, Osaka, Japan), Atsunori Hashimoto (Hyogo College of Medicine, Nishinomiya, Japan), Hiroyuki Koami (Saga University Hospital, Saga, Japan), Satoru Beppu (National Hospital Organization Kyoto Medical Center, Kyoto, Japan), Yoichi Katayama (Sapporo Medical University, Sapporo, Japan), and Makoto Itoh (Yamaguchi Grand Medical Center, Yamaguchi, Japan).

\section{Disclosure Statement}

We declare that we have no competing interests in this study.

\section{Funding Source}

The DESIRE trial was supported, in part, by a non-contractual research grant from Hospira Japan to Wakayama Medical University. 


\section{References}

1 Angus DC, Linde-Zwirble WT, Lidicker J, Clermont G, Carcillo J, Pinsky MR: Epidemiology of severe sepsis in the United States: analysis of incidence, outcome, and associated costs of care. Crit Care Med 2001;29:13031310 .

2 Novelli G, Ferretti G, Poli L, Pretagostini R, Ruberto F, Perrella SM, Levi Sandri GB, Morabito V, Berloco PB: Clinical results of treatment of postsurgical endotoxin-mediated sepsis with polymyxin-B direct hemoperfusion. Transplant Proc 2010;42:1021-1024.

3 Camussi G, Ronco C, Montrucchio G, Piccoli G: Role of soluble mediators in sepsis and renal failure. Kidney Int Suppl 1998;66:S38S42.

4 Ronco C, Brendolan A, Dan M, Piccinni P, Bellomo R, De Nitti C, Inguaggiato P, Tetta C: Adsorption in sepsis. Kidney Int Suppl 2000; 76:S148-S155.

5 Bellomo R, Ronco C: Blood purification in the intensive care unit: evolving concepts. World J Surg 2001;25:677-683.

6 Ronco C, Ricci Z, De Backer D, Kellum JA, Taccone FS, Joannidis M, Pickkers P, Cantaluppi V, Turani F, Saudan P, Bellomo R, Joannes-Boyau O, Antonelli M, Payen D, Prowle JR, Vincent JL: Renal replacement therapy in acute kidney injury: controversy and consensus. Crit Care. 2015;19:146.

7 Zhou F, Peng Z, Murugan R, Kellum JA: Blood purification and mortality in sepsis: a meta-analysis of randomized trials. Crit Care Med 2013;41:2209-2220.

8 Chang T, Tu YK, Lee CT, Chao A, Huang CH, Wang MJ, Yeh YC: Effects of polymyxin B hemoperfusion on mortality in patients with severe sepsis and septic shock: a systemic review, meta-analysis update, and disease severity subgroup meta-analysis. Crit Care Med 2017;45:e858-e864.

9 Payen DM, Guilhot J, Launey Y, Lukaszewicz AC, Kaaki M, Veber B, Pottecher J, Joannes-
Boyau O, Martin-Lefevre L, Jabaudon M, Mimoz O, Coudroy R, Ferrandière M, Kipnis E, Vela C, Chevallier S, Mallat J, Robert R; ABDOMIX Group: Early use of polymyxin B hemoperfusion in patients with septic shock due to peritonitis: a multicenter randomized control trial. Intensive Care Med 2015;41:975984.

10 Oda S, Aibiki M, Ikeda T, Imaizumi H, Endo S, Ochiai R, Kotani J, Shime N, Nishida O, Noguchi T, Matsuda N, Hirasawa H, and Sepsis Registry Committee of JSICM: The Japanese guidelines for the management of sepsis. J Intensive Care 2014;2:55.

11 Kawazoe Y, Miyamoto K, Morimoto T, Yamamoto T, Fuke A, Hashimoto A, Koami H, Beppu S, Katayama Y, Itoh M, Ohta Y, Yamamura H; Dexmedetomidine for Sepsis in Intensive Care Unit Randomized Evaluation (DESIRE) Trial Investigators: Effect of dexmedetomidine on mortality and ventilatorfree days in patients requiring mechanical ventilation with sepsis: a randomized clinical trial. JAMA 2017;317:1321-1328.

12 Cruz DN, Perazella MA, Bellomo R, de Cal M, Polanco N, Corradi V, Lentini P, Nalesso F, Ueno T, Ranieri VM, Ronco C: Effectiveness of polymyxin B-immobilized fiber column in sepsis: a systematic review. Crit Care 2007; 11:R47.

13 Cruz DN, Antonelli M, Fumagalli R, Foltran F, Brienza N, Donati A, Malcangi V, Petrini F, Volta G, Bobbio Pallavicini FM, Rottoli F, Giunta F, Ronco C: Early use of polymyxin B hemoperfusion in abdominal septic shock: the EUPHAS randomized controlled trial. JAMA 2009;301:2445-2452.

14 Shoji H: Extracorporeal endotoxin removal for the treatment of sepsis: endotoxin adsorption cartridge (Toraymyxin). Ther Apher Dial 2003; 7:108-114.

15 Mitaka C, Fujiwara N, Yamamoto M, Toyofuku T, Haraguchi G, Tomita M: Polymyxin
B-immobilized fiber column hemoperfusion removes endotoxin throughout a 24-hour treatment period. J Crit Care 2014;29:728732 .

16 Mitaka C, Tsuchida N, Kawada K, Nakajima Y, Imai T, Sasaki S: A longer duration of polymyxin B-immobilized fiber column hemoperfusion improves pulmonary oxygenation in patients with septic shock. Shock 2009;32: 478-483.

17 Yamashita C, Hara Y, Kuriyama N, Nakamura T, Nishida O: Clinical effects of a longer duration of polymyxin B-immobilized fiber column direct hemoperfusion therapy for severe sepsis and septic shock. Ther Apher Dial 2015;19:316-323.

18 Miyamoto K, Kawazoe Y, Kato S: Prolonged direct hemoperfusion using a polymyxin $\mathrm{B}$ immobilized fiber cartridge provides sustained circulatory stabilization in patients with septic shock: a retrospective observational before-after study. J Intensive Care 2017;5:19.

19 Kawamata $T$, Imaizumi $H$, Yoshida $M$, Kaneko M: Polymyxin B-immobilized fiber improves hyperdynamic state in MRSA septic patients. Intensive Care Med 1997;23:130131

20 Danner RL, Elin RJ, Hosseini JM, Wesley RA, Reilly JM, Parillo JE: Endotoxemia in human septic shock. Chest 1991;99:169-175.

21 Opal SM: The clinical relevance of endotoxin in human sepsis: a critical analysis. J Endotoxin Res 2002;8:473-476.

22 Wang Y, Liu Y, Sarker KP, Nakashima M, Serizawa T, Kishida A, Akashi M, Nakata M, Kitajima I, Maruyama I: Polymyxin B binds to anandamide and inhibits its cytotoxic effect. FEBS Lett 2000;470:151-155.

23 Spectral Medical Inc., Advancing a Novel Treatment for Septic Shock (cited July 31, 2017). http://www.spectraldx.com/assets/ spectral_investor_update_0717.pdf. 